\title{
An Integral Representation for the Polylogarithm Function and Some Special Values
}

\section{Edigles Guedes ${ }^{1}$ and Prof. Dr. K. Raja Rama Gandhi ${ }^{2}$ \\ Number Theorist, Brazil ${ }^{1}$}

Resource perosn in Mathematics for Oxford University Press and Professor at BITS-Vizag ${ }^{2}$

Abstract. I proved a new integral representation for the polylogarithm function.

\section{INTRODUCTION}

Using an integral representation and unorthodox method of substitution, I demonstrated that:

$$
\operatorname{Li}_{s}(z)=\frac{z}{\Gamma(s)} \int_{0}^{\infty} \frac{[\ln (1+u)]^{s-1}}{(1+u)(1+u-z)} d u .
$$

\section{THEOREM}

Theorem 1. For $\mathfrak{R}(z)<1$ or $\mathfrak{I}(z) \neq 0$, then

$$
\operatorname{Li}_{s}(z)=\frac{z}{\Gamma(s)} \int_{0}^{\infty} \frac{[\ln (1+u)]^{s-1}}{(1+u)(1+u-z)} d u,
$$

where $\mathrm{Li}_{s}(z)$ denotes the polylogarithm function, $\Gamma(s)$ denotes the gamma function and $\ln s$ denotes the natural logarithm.

Proof. In [1], the polylogarithm function is defined by

$$
\operatorname{Li}_{S}(z):=\frac{z}{\Gamma(s)} \int_{0}^{1} \frac{[\ln (1 / t)]^{s-1}}{1-z t} d t
$$

for $\mathfrak{R}(s)>0$.

Take $t=\frac{a+b u}{1+u}$ and $d t=\left[\frac{b}{1+u}-\frac{a+b u}{(1+u)^{2}}\right] d u$ in (2), I obtain

$$
\operatorname{Li}_{S}(z):=\frac{z}{\Gamma(s)} \int_{-\frac{a}{b}}^{-\frac{a-1}{b-1}}\left[\frac{\left[\ln \left(\frac{1+u}{a+b u}\right)\right]^{S-1}}{1-z \frac{a+b u}{1+u}}\right]\left[\frac{b}{1+u}-\frac{a+b u}{(1+u)^{2}}\right] d u
$$

Let $-\frac{a-1}{b-1}=\tan (\pi \theta)$ and $-\frac{a}{b}=\cot (\pi \theta)$, therefore,

$$
a=\frac{(\tan (\pi \theta)+1) \cot (\pi \theta)}{\cot (\pi \theta)-\tan (\pi \theta)}, \quad b=-\frac{\tan (\pi \theta)+1}{\cot (\pi \theta)-\tan (\pi \theta)} .
$$

Put (4) in (3) and simplifying, I have

$$
\operatorname{Li}_{s}(z)=\frac{z}{\Gamma(s)} \int_{\cot (\pi \theta)}^{\tan (\pi \theta)} f(u, \theta) d u
$$

where

$$
f(u, \theta)=\frac{(-\cot (\pi \theta)-1) \ln ^{s-1}\left(-\frac{(u+1)(\cot (\pi \theta)-1)}{u-\cot (\pi \theta)}\right)}{(u+1)(\cot (\pi \theta)(u-z+1)+u(z-1)-1)}
$$


Consider the limit $\theta \rightarrow 1$ in (5)

$$
\lim _{\theta \rightarrow 1} \operatorname{Li}_{s}(z)=\frac{z}{\Gamma(s)} \lim _{\theta \rightarrow 1} \int_{\cot (\pi \theta)}^{\tan (\pi \theta)} f(u, \theta) d u,
$$

then

$$
\begin{gathered}
\lim _{\theta \rightarrow 1} \operatorname{Li}_{s}(z)=\operatorname{Li}_{s}(z), \\
\lim _{\theta \rightarrow 1} f(u, \theta)=-\frac{\ln (1+u)^{s-1}}{(1+u)(1+u-z)}, \\
\lim _{\theta \rightarrow 1} \tan (\pi \theta)=0, \\
\lim _{\theta \rightarrow 1} \cot (\pi \theta)=\infty .
\end{gathered}
$$

Hence, from (6) and (7), it follows that

$$
\operatorname{Li}_{s}(z)=\frac{z}{\Gamma(s)} \int_{0}^{\infty} \frac{\ln (1+u)^{s-1}}{(1+u)(1+u-z)} d u .
$$

Corollary 1. For $\mathfrak{R}(z)<1$ or $\mathfrak{I}(z) \neq 0$, then

$$
6 \mathrm{Li}_{2}(z)=-\pi^{2}+3 \ln ^{2}(1-z)-6 \ln (1-z) \ln (-z)+6 \mathrm{Li}_{2}\left(\frac{1}{1-z}\right),
$$

where $\mathrm{Li}_{2}(z)$ denotes the dilogarithm function and $\ln z$ denotes the natural logarithm.

Proof. I let $s=2$ in Theorem 1 .

Special Values. Let $z=\frac{1}{2}$ in Corollary 1 , then

$$
6 \mathrm{Li}_{2}\left(\frac{1}{2}\right)=-\pi^{2}+3 \ln ^{2}\left(\frac{1}{2}\right)-6 \ln \left(\frac{1}{2}\right) \ln \left(-\frac{1}{2}\right)+6 \mathrm{Li}_{2}(2) .
$$

On the other hand, in [2], I find

$$
\operatorname{Li}_{2}\left(\frac{1}{2}\right)=\frac{\pi^{2}}{12}-\frac{\ln ^{2} 2}{2} .
$$

I substitute (10) in the left hand side of the (9), and encounter

$$
\mathrm{Li}_{2}(2)=\frac{\pi^{2}}{4}-\mathrm{i} \pi \ln 2 \text {. }
$$

Let $z=\frac{2}{3}$ in Corollary 1 , then

$$
\mathrm{Li}_{2}(3)-\mathrm{Li}_{2}\left(\frac{2}{3}\right)=\frac{\pi^{2}}{6}+\frac{\ln ^{2} 3}{2}-\ln 2 \ln 3-\mathrm{i} \pi \ln 3 .
$$

Let $z=\frac{3}{4}$ in Corollary 1 , then

$$
\mathrm{Li}_{2}(4)-\mathrm{Li}_{2}\left(\frac{3}{4}\right)=\frac{\pi^{2}}{6}+2 \ln ^{2} 2-2 \ln 2 \ln 3-2 \text { i } \pi \ln 2 .
$$


From (13), I conclude that

$$
\begin{aligned}
& \operatorname{Li}_{2}(4)=2 \cdot{ }_{3} F_{2}\left(\begin{array}{c|c}
\frac{1}{2}, \frac{1}{2}, 1 & 1 \\
\frac{3}{2}, \frac{3}{2} & \frac{1}{4}
\end{array}\right)-2 \mathrm{i} \pi \ln 2 \\
= & \frac{\pi^{2}}{6}-\ln ^{2} 2-2 \operatorname{Li}_{2}\left(-\frac{1}{2}\right)-2 \mathrm{i} \pi \ln 2
\end{aligned}
$$

and

$$
\mathrm{Li}_{2}\left(\frac{3}{4}\right)=2 \ln 2 \ln 3-3 \ln ^{2} 2-2 \mathrm{Li}_{2}\left(-\frac{1}{2}\right)
$$

Let $z=\frac{4}{5}$ in Corollary 1 , then

$$
\mathrm{Li}_{2}(5)-\mathrm{Li}_{2}\left(\frac{4}{5}\right)=\frac{\pi^{2}}{6}+\frac{\ln ^{2} 5}{2}-2 \ln 2 \ln 5-\mathrm{i} \pi \ln 5,
$$

Let $z=\frac{5}{6}$ in Corollary 1 , then

$$
\mathrm{Li}_{2}(6)-\mathrm{Li}_{2}\left(\frac{5}{6}\right)=\frac{\pi^{2}}{6}+\frac{\ln ^{2} 6}{2}-\ln 5 \ln 6-\mathrm{i} \pi \ln 6
$$

Theorem 2. For $\mathfrak{R}(s)>1$, then

$$
\mathrm{Li}_{2}(z)+\mathrm{Li}_{2}\left(\frac{1}{z}\right)=\frac{\pi^{2}}{3}-\frac{\ln ^{2} z}{2}-\mathrm{i} \pi \ln z,
$$

where $\mathrm{Li}_{S}(z)$ denotes the polylogarithm function and $\ln s$ denotes the natural logarithm.

Proof. In Theorem 1, the polylogarithm function is defined by

$$
\operatorname{Li}_{S}(z):=\frac{z}{\Gamma(s)} \int_{0}^{\infty} \frac{[\ln (1+u)]^{s-1}}{(1+u)(1+u-z)} d u
$$

for $\mathfrak{R}(s)>0$.

Let $s=2$; thus, the dilogarithm function is

$$
\mathrm{Li}_{2}(z)=z \int_{0}^{\infty} \frac{\ln (1+u)}{(1+u)(1+u-z)} d u
$$

On the other hand, in [3], I prove that

$$
\ln z=(z-1) \int_{0}^{\infty} \frac{1}{(t+z)(t+1)} d t
$$

for $\Re(z)>0$.

From (20) and (21), it follows that

$$
\operatorname{Li}_{2}(z)=z \int_{0}^{\infty} \frac{u}{(1+u)(1+u-z)} \int_{0}^{\infty} \frac{1}{(1+u+t)(1+t)} d t d u
$$




$$
\begin{aligned}
& =z \int_{0}^{\infty} \frac{1}{1+t} \int_{0}^{\infty} \frac{u}{(1+u)(1+u+t)(1+u-z)} d u d t \\
= & z \int_{0}^{\infty} \frac{1}{t+1} \frac{(t+1) z \ln (t+1)-t(z-1) \ln (1-z)}{t z(t+z)} d t \\
= & z \int_{0}^{\infty} \frac{\ln (t+1)}{t(t+z)} d t-(z-1) \ln (1-z) \int_{0}^{\infty} \frac{1}{(t+1)(t+z)} d t \\
= & \frac{2 \pi^{2}+6 \ln (z-1) \ln z-3 \ln ^{2} z-6 \operatorname{Li}_{2}\left(\frac{1}{z}\right)}{6}-\ln (1-z) \ln z \\
= & \frac{\pi^{2}}{3}+[\ln (z-1)-\ln (1-z)] \ln z-\frac{\ln ^{2} z}{2}-\operatorname{Li}_{2}\left(\frac{1}{z}\right),
\end{aligned}
$$

ergo,

$$
\operatorname{Li}_{2}(z)+\operatorname{Li}_{2}\left(\frac{1}{z}\right)=\frac{\pi^{2}}{3}-\frac{\ln ^{2} z}{2}-\mathrm{i} \pi \ln z
$$

Theorem 2. For $\mathfrak{R}(s)>1$, then

$$
\mathrm{Li}_{2}(z)+\mathrm{Li}_{2}\left(\frac{1}{z}\right)=\frac{\pi^{2}}{3}-\frac{\ln ^{2} z}{2}-\mathrm{i} \pi \ln z,
$$

where $\mathrm{Li}_{s}(z)$ denotes the polylogarithm function and $\ln s$ denotes the natural logarithm.

Proof. In Theorem 1, the polylogarithm function is defined by

$$
\operatorname{Li}_{s}(z):=\frac{z}{\Gamma(s)} \int_{0}^{\infty} \frac{[\ln (1+u)]^{s-1}}{(1+u)(1+u-z)} d u,
$$

for $\mathfrak{R}(s)>0$.

Let $s=2$; thus, the dilogarithm function is

$$
\mathrm{Li}_{2}(z)=z \int_{0}^{\infty} \frac{\ln (1+u)}{(1+u)(1+u-z)} d u
$$

On the other hand, in [3], I prove that

$$
\frac{\ln x}{x-1}=\int_{0}^{1} \frac{t^{-1}}{(\ln t-x)(\ln t-1)} d t
$$

for $\mathfrak{R}(z)>1$. I set $x=1+u$ in (26), and obtain

$$
\ln (1+u)=u \int_{0}^{1} \frac{t^{-1}}{(\ln t-u-1)(\ln t-1)} d t
$$

From (25) and (27), it follows that

$$
\begin{gathered}
\operatorname{Li}_{2}(z)=z \int_{0}^{\infty} \frac{u}{(1+u)(1+u-z)} \int_{0}^{1} \frac{t^{-1}}{(\ln t-u-1)(\ln t-1)} d t d u \\
=z \int_{0}^{1} \frac{t^{-1}}{\ln t-1} \int_{0}^{\infty} \frac{u}{(1+u)(1+u-z)(\ln t-u-1)} d u d t
\end{gathered}
$$




$$
\begin{array}{r}
=z \int_{0}^{\infty} \frac{1}{t+1} \frac{(t+1) z \ln (t+1)-t(z-1) \ln (1-z)}{t z(t+z)} d t \\
=z \int_{0}^{\infty} \frac{\ln (t+1)}{t(t+z)} d t-(z-1) \ln (1-z) \int_{0}^{\infty} \frac{1}{(t+1)(t+z)} d t \\
=\frac{2 \pi^{2}+6 \ln (z-1) \ln z-3 \ln ^{2} z-6 \operatorname{Li}_{2}\left(\frac{1}{z}\right)}{6}-\ln (1-z) \ln z \\
=\frac{\pi^{2}}{3}+[\ln (z-1)-\ln (1-z)] \ln z-\frac{\ln ^{2} z}{2}-\operatorname{Li}_{2}\left(\frac{1}{z}\right),
\end{array}
$$

ergo,

$$
\operatorname{Li}_{2}(z)+\operatorname{Li}_{2}\left(\frac{1}{z}\right)=\frac{\pi^{2}}{3}-\frac{\ln ^{2} z}{2}-\mathrm{i} \pi \ln z
$$

\section{REFERENCES}

[1] Cvijovic, Djurdje, New integral representations of the polylogarithm function, Proc. R. Soc. A 2007 463, doi: 10.1098/rspa.2006.1794, published 8 April 2007:

available in http://rspa.royalsocietypublishing.org/content/463/2080/897.full.pdf.

[2] http://en.wikipedia.org/wiki/Polylogarithm, available in March 5, 2014.

[3] Guedes, Edigles, The Natural Logarithm and its Applications, to appear, 2014. 